\title{
Estudio de las temáticas y contenidos de las tesis doctorales realizadas en España sobre Biomecánica Deportiva Study of the themes and contents of the doctoral theses carried out in Spain on Sports Biomechanics

\author{
*José Campos Granell, ** Marcos Gutiérrez-Dávila, *** José María Campos Coll \\ *Universidad de Valencia (España), ${ }^{* *}$ Universidad de Granada (España), ${ }^{* * *}$ I.E.S. Districte Maritim. Valencia (España)
}

Resumen: El objeto del estudio es conocer y valorar la evolución de las temáticas y contenidos de las tesis doctorales que se han leído en España sobre Biomecánica Deportiva durante el periodo comprendido entre los años 1980 y 2019. La muestra está compuesta de 233 tesis después de un proceso de selección con el uso de criterios de inclusión/exclusión y términos de búsqueda específicos. Los resultados demuestran que existe un crecimiento progresivo en la producción de tesis doctorales a lo largo del tiempo con incrementos progresivos, especialmente en el tercer y cuarto periodo. Los campos temáticos con mayor número de tesis leídas son los de Biomecánica de Apoyo y de Biomecánica Aplicada. El campo de Biomecánica Básica resulta residual, con tres tesis leídas a lo largo del periodo investigado. En la Biomecánica de Apoyo, el subcampo más representado y con un crecimiento constante, es el del Área Médica, con 73 tesis leídas que representa el 68,2\% del total del campo temático. En la Biomecánica Aplicada, los subcampos con más tesis leídas son los de Análisis de la Técnica Deportiva y de Análisis del Movimiento con 35 y 32 tesis leídas, respectivamente, sin que se observe un crecimiento constante a lo largo del tiempo. El campo de Biomecánica-Tecnologías es el tercero con un 10,7\% de las tesis leídas. El término más utilizado en los títulos de las tesis es el de la «marcha» que se convierte en un tema transversal que ha sido abordado desde diferentes perspectivas científicas.

Palabras clave: Investigación documental, tesis doctorales, biomecánica deportiva, investigación, deporte.

\begin{abstract}
The purpose of the study is to know and assess the evolution of the themes and contents of the doctoral theses that have been read in Spain on Sports Biomechanics during the period between 1980 and 2019. The sample is made up of 233 theses after a selection process with the use of inclusion / exclusion criteria and specific search terms. The results show that there is a progressive growth in the production of doctoral theses over time with progressive increases, especially in the third and fourth periods. The thematic fields with the highest number of theses read are Support Biomechanics and Applied Biomechanics. The Basic Biomechanics field is a residual field with 3 theses read over time. In Support Biomechanics, the most represented subfield with constant growth is the Medical Area with 73 theses read, representing $68.2 \%$ of the total thematic field. In Applied Biomechanics, the subfields with the most theses read are the Analysis of Sports Technique and Analysis of Movement with 35 and 32 theses read, with no constant growth observed over time. The Biomechanics-Technologies field is the third with $10.7 \%$ of the theses read. The term most used in the thesis titles is «walking», which becomes a cross-cutting theme that has been approached from different scientific perspectives.
\end{abstract}

Keywords: documentary research, doctoral theses, sport biomechanics, research, sport.

\section{Introducción}

Las Tesis Doctorales representan una herramienta útil para evaluar el estado de la investigación y, través de sus contenidos, conocer las tendencias que la orientan permitiendo de esta manera, una reconstrucción de la evolución de cualquier área de conocimiento.

En España, el desarrollo de la Biomecánica deportiva ha sido tardío en comparación con el proceso seguido en otros países. Sin embargo, en las últimas décadas se ha producido un avance extraordinario gracias a la

Fecha recepción: 28-07-21. Fecha de aceptación: 20-11-21

Jose Campos Granel

Jose.Campos@uv.es actividad desarrollada en los laboratorios de los Departamentos de Educación Física y Deporte de los INEFs, de las Facultades de Ciencias de la Actividad Física y del Deporte (CAFD) y también de otros Centros y Departamentos en los que a pesar de no impartir el título de Licenciado en CAFD, han mantenido líneas de investigación y actividad continuada en esta materia. Sirva como muestra del dinamismo de este campo de investigación que, entre los proyectos de investigación financiados por la Comisión Interministerial de Ciencia y Tecnología (CICyT) durante los años 1988 y 1994, el área de Biomecánica se situaba en tercer lugar, con un 5,5\% del total de la financiación ofertada, por detrás de la Medicina, que era la mayoritaria con un $69,4 \%$ y de la de Psicología con un 6,7\% (Pastor Pradillo, 2003). 
También, los avances tecnológicos han contribuido al desarrollo de este campo de investigación gracias a la aparición de dispositivos e instrumentos de medida y control que han permitido utilizar protocolos de intervención más complejos y fiables. Instrumentos que, además, han propiciado la creación de nuevas patentes y con ellas el desarrollo tecnológico y avance en el conocimiento acerca de esta disciplina.

Tradicionalmente, la Biomecánica Deportiva ha centrado su campo de estudio en el análisis de la técnica deportiva en su orientación al apoyo del entrenamiento de los deportistas. Sin embargo, la Biomecánica Deportiva ha evolucionado de forma notable ampliando su campo de acción. En la actualidad, hace referencia a un marco más amplio donde a los especialistas en la Biomecánica Deportiva se les han añadido profesionales procedentes de disciplinas diversas como los técnicos deportivos, pedagogos, psicólogos, médicos, ingenieros, fisiólogos, o fisioterapeutas, con el objetivo de abordar en mejores condiciones el estudio de los sistemas que gobiernan las técnicas deportivas, el movimiento humano, la salud y la calidad de vida de ciudadanos y deportistas.

Como muestra de esta forma de colaboración profesional, sirva como ejemplo la estructura en que está configurada la Sociedad Ibérica de Biomecánica y Biomateriales. Una sociedad creada en el año 1977 y en la que se integran tres ámbitos de estudio como son: el de Biomateriales, vinculado al campo de las Ingenierías, el grupo de Biomecánica Médica, en el que participan traumatólogos y cirujanos ortopédicos, y el ámbito de estudio de la Biomecánica Deportiva en el que participan profesionales de disciplinas diversas vinculadas, directa, o indirectamente, a las Ciencias de la Actividad Física y del Deporte.

Como una referencia de los ámbitos en los que se desarrolla y avanza la investigación en Biomecánica Deportiva, las áreas que integran los contenidos en Congresos y Jornadas Científicas constituyen una información valiosa. Como ejemplo de ello, la XXXIV International Conference of Biomechanics in Sport celebrada en el año 2016 en Tsukuba (Japón) (Michiyoshi Ae,Yasushi Enomoto, Norihisa Fujii, HidekiTakagi, 2016). En dicho congreso se establecieron siete áreas para presentar trabajos de investigación: 1) Methodology, 2) Modelling/Simulation, 3) Muscle-Skeleton-Mechanics, 4) Injuries/Rehabilitation, Equipment/Instrumentation, 5) Coaching and Sport Activities, y 6) Applied Program. De ellas, las más representadas fueron, en primer lugar y de forma destacada, el área de Coaching and Sport
Activities en la que se presentaron 157 trabajos, lo que suponía el 51\% del total y en segundo lugar, el de Injuries/Rehabilitation, en la que se presentaron 74 trabajos, lo que suponía el 24\% del total. En suma, un programa en el que la investigación aplicada al entrenamiento y a la actividad física fueron la referencia principal, seguida de la orientada al estudio de las lesiones y la rehabilitación dentro del ámbito sanitario

De la revisión realizada en la literatura se han encontrado escasas referencias de estudios dedicados a la evolución y análisis de contenido de tesis doctorales realizadas en el campo de la Actividad Física y del Deporte, sin embargo, no tenemos constancia de la existencia de trabajos orientados a la evolución de tesis doctorales realizadas en el campo específico de la Biomecánica de la Actividad Física y del Deporte, ni tampoco del análisis de sus contenidos y de los campos de investigación que abordan.

La información disponible sobre el análisis y evolución de las Tesis Doctorales procede de estudios globales, que han sido realizados en el ámbito de las Ciencias de la Actividad Física y el Deporte, como es el caso del estudio de Delgado \& Medina, (1997). En el análisis que realizan estos autores se establece que, dentro del Área de las Ciencias Biomédicas, la Biomecánica de las Técnicas Deportivas representa el 9,9\% del total de las tesis leídas. Cabe señalar también dos estudios más, el realizado por Ponce de León, Gargallo y Loza (1998) donde se analizan las tendencias en las tesis doctorales de Educación Física entre los cursos 1980-81 y 1995-96 y el realizado por García García (2016) sobre la evolución de las tesis doctorales en Educación Física y Deportes entre los años 1990 y 2013, aunque en ambos casos no se ofrece información sobre el campo de la Biomecánica Deportiva, ni de sus contenidos u objetos de investigación.

Con todo, el objetivo del presente estudio está centrado en analizar la evolución de las temáticas y contenidos de las tesis doctorales realizadas en el campo específico de la Biomecánica Deportiva durante el periodo comprendido entre los años 1980 y 2019. Se trata pues, de un análisis sincrónico de la producción científica vinculada a Tesis Doctorales en sus diferentes fases, tanto de carácter cuantitativo, como cualitativo.

\section{Material y Método}

\section{Muestra}

Después de un proceso de búsqueda y selección que se concreta en el apartado del Procedimiento, el estu- 
dio se realiza sobre una muestra de 233 tesis doctorales, leídas en Universidades Españolas, tanto públicas como privadas, durante el periodo comprendido entre los cursos académicos 1980-81 y 2018-19.

\section{Procedimientos}

Para la localización de las tesis doctorales se utilizó la base de datos TESEO del Ministerio de Educación, Cultura y Deporte, y de forma complementaria, la base de datos DIALNET. En el proceso de búsqueda y dado que, en una primera fase de búsqueda en TESEO, se encontraron registros en los que no constaban datos del programa de doctorado, ni de los Departamentos Universitarios, se realizó una búsqueda complementaria en las bases de datos de las Universidades en las que se leyeron las tesis. Dicha búsqueda permitió reducir a tres el número de tesis excluidas por no haber incluido el Departamento responsable.

Para el almacenamiento y organización de la información se utilizó el programa EXCEL 2013. Posteriormente, se trasladaron los datos al programa estadístico SPSS V21 para su tabulación, codificación de las variables del estudio y obtención de los resultados.

Para la selección de la muestra se utilizaron dos criterios de inclusión: a) que las tesis hubieran sido leídas entre los cursos académicos 1980-81 y 2018-19 y b), que el tema central de las mismas estuviera vinculado con la Biomecánica en el campo específico de la Actividad Física y el Deporte. Se excluyeron, por tanto, las tesis que se hubieran realizado fuera de este campo para lo que se revisaron, no solo los títulos de las tesis, sino también sus resúmenes.

En la base de datos TESEO se utilizaron 32 términos de búsqueda, específicamente vinculados a la Biomecánica Deportiva y todos sus ámbitos de estudio. Concretamente se utilizaron los siguientes términos de búsqueda: Biomecánica; Biomecánica y deporte; Biomecánica y educación física; Biomecánica y actividad física; Ergonomía; Cinemática; Biomecánica de la columna; Biomecánica del pie; Pie diabético; control postural; Electromiografía; Pedobarografía; Plantillas; Isocinético; Análisis Biomecánico; Fotogrametría; Dinámica Inversa; Valoración biomecánica; Calzado; Cinético; Cinética; Marcha; SaltoVertical; Contramovimiento;Biomecánico; Cinemático; plataforma de fuerzas;Tridimensional;Tridimensional y deporte; Rendimiento deportivo; Rendimiento muscular, y Anticipación.

Posteriormente, una vez registradas las tesis que cumplían con los criterios de selección establecidos, se pasó a eliminar los registros duplicados. Complementariamente, para confirmar y completar datos que no se ofrecían en la base TESEO, se realizaron búsquedas en DIALNET a partir de los nombres de 23 investigadores que son referentes en España en el campo de la Biomecánica Deportiva.

En la figura 1 se presenta un diagrama de flujo que representa el proceso de selección de la muestra final del estudio en sus diferentes fases y la aplicación de los criterios de inclusión/exclusión fijados para realizar la selección de la muestra final que quedó fijada en un total de 233 tesis doctorales.

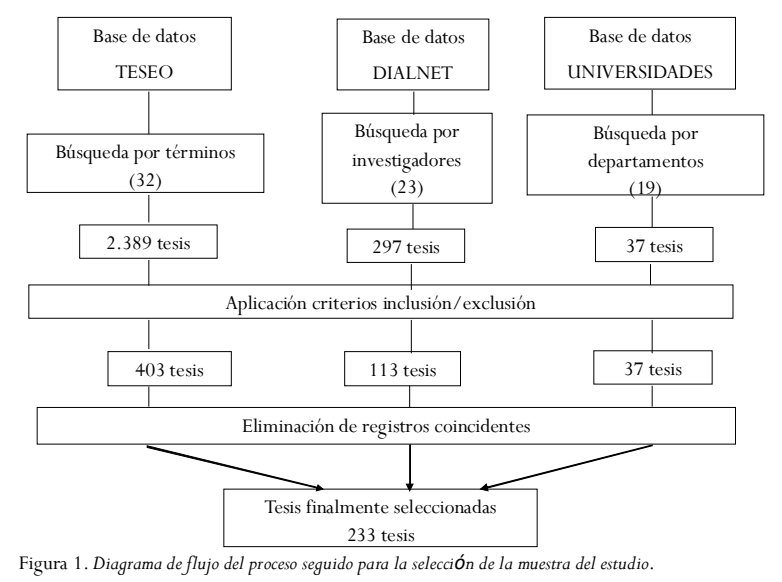

Variables del estudio y tratamiento estadístico

En el presente trabajo se presentan datos referidos a las siguientes variables: Año/periodo de lectura, Campos temáticos, Subcampos temáticos.

Para el tratamiento de los datos se utilizó el paquete estadístico SPSS V21, a partir del cálculo de descriptivos (frecuencias y porcentajes), y de tablas de contingencia con análisis de residuos tipificados corregidos (Haberman, 1973) con la utilización de un contraste basado en el estadístico Chi-cuadrado $(\chi 2)$ para identificar relaciones de dependencia y fijando como valor de significación estadística $p \leq .05$.

En relación con la temática y contenidos de las tesis se establecieron los siguientes campos temáticos:

Biomecánica básica. Teorías explicativas del movimiento humano y deportivo.

Biomecánica aplicada. Trabajos cuya variable independiente es del ámbito de la biomecánica, estableciéndose tres subgrupos: 1 / Análisis de la técnica deportiva, 2/ Análisis del movimiento humano, 3/ Análisis varios, como trabajos sobre la fuerza muscular, sobre la coordinación del movimiento, desarrollo y elaboración de test de fuerza o coordinación, etc

Biomecánica de apoyo. Vinculada a las perspectivas científicas de ámbito deportivo. Trabajos cuya variable independiente y objetivo concreto que se utiliza no responde a ninguna variable biomecánica, pero 
que la utilizan como medio para alcanzar los objetivos. Cuando se evalúa un entrenamiento concreto, un programa para la salud, una variable sociológica, conductual, psicológica etc. Se establecieron cuatro subcampos temáticos: 1/ Fisiología; 2/ Control Motor; 3 / Área Médica; 4/ Otros (Sociología/Gestión; materiales, ergonomía, vestuario, calzado, confort, instalaciones, ...)

Biomecánica-tecnología.Trabajos orientados en los desarrollos biomecánicos que pueden utilizarse para registrar el movimiento, incluida su causa. Se establecieron tres subcampos temáticos: 1/ Fotogrametría; 2/ Dinamometría/Acelerometría; 3/ Otras tecnologías (termografía, modelado, sensores inerciales, realidad virtual, robótica, resonancia magnética, plantillas instrumentadas, etc).

Por otro lado, también se utilizó como elemento de clasificación el periodo en que las tesis fueron leídas en sus respectivas Universidades. Para fijar los períodos académicos se utilizaron criterios vinculados a cuestiones normativas y de ocurrencia de determinados acontecimientos que hubieran marcado la evolución de los Centros y de los Programas de Doctorado en el campo de la Actividad Física y el Deporte en España. Concretamente, se establecieron los siguientes periodos académicos:

Periodo de normalización académica. Comprendido entre los cursos 1980/81 y 1989/ 90: Se aprueba la Ley Cultura Física y el Deporte de 1980. En 1981, el Real Decreto 790/1981 aprueba una nueva regulación de los INEFs ampliando a cinco años y reconocimiento de la equivalencia a titulaciones universitarias. En el curso 1988-89, se produce la aprobación y aplicación del Tercer Ciclo. Entre los años 198689 se ponen en marcha los primeros Programas de Doctorado desde Departamentos externos a los INEFs, como fueron los casos de la Universidad Complutense de Madrid, el Departamento de Personalidad, Evaluación y Tratamiento Psicológico de la Universidad de Granada y el Departamento de Teoría e Historia de la Educación de la Universitat de Barcelona. En suma, un periodo donde existían importantes dificultades legales para leer las tesis doctorales por parte de los Licenciados en Educación Física (EF).

Periodo de adaptación al paradigma Universitario. Comprendido entre los cursos 1990/ 91 y 1999/00: Ya hay doctores procedentes de los primeros Programas de Doctorado de Licenciados y Titulados en EF/CAFD. Inicio de proyectos de investigación financiados en los Planes Nacionales de Investigación I+D+i. El BOE 17 de noviembre de 1994 publica el Real Decreto de incorporación del título de Licenciado en Educación Física al catálogo de títulos oficiales válidos en todo el estado. Se presenta el Libro Blanco de la I+D en el Deporte. El 15 de octubre de 1998 ese mismo año se constituye la Asociación Española de Ciencias del Deporte.

Periodo de consolidación Universitaria de los estudios. Comprendido entre los cursos 2000/01 y 2009/10: Como consecuencia del Proceso de Bolonia para la integración al Espacio Europeo de Educación Superior, se implanta en España el nuevo título de Grado en CAFD. Supone un periodo de integración y reconocimiento académico en el que se produce un significativo desarrollo y crecimiento de la producción científica en las Facultades y Centros en Ciencias de la Actividad Física y el Deporte.

Periodo de internacionalización. Comprendido entre los cursos 2010/11 y 2018/19: Etapa de internacionalización de los Centros y Facultades Españolas, así como del reconocimiento del potencial investigador de las Facultades, Departamentos y Grupos de Investigación de todo el Estado, así como su participación en Programas Europeos.

\section{Resultados}

\section{Las tesis doctorales en Biomecánica Deportiva} y su evolución en el tiempo

En la tabla 1, se presenta la distribución de las tesis leídas en cada uno de los periodos académicos establecidos, comprobándose que existe un crecimiento progresivo en la producción de tesis doctorales a lo largo del tiempo con incrementos progresivos, especialmente en el tercer y cuarto periodo, y que vienen a demostrar que fueron los más productivos, especialmente el último periodo entre los cursos 2010-2019, con un total de 109 tesis leídas, lo que representa el 46,9\% del total, esto es, prácticamente la mitad de la tesis doctorales leídas desde el año 1980. Por el contrario, y como era de esperar, el primer periodo resultó ser en el que se leyeron el menor número de tesis, tan sólo 10, lo que representa el 4,3\% del total.

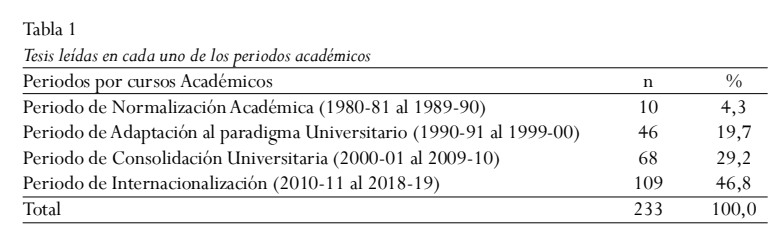

Las temáticas y contenidos de las Tesis

En la tabla 2 se presentan las tesis leídas a lo largo de los cuatro periodos académicos en cada uno de los cam- 
pos temáticos establecidos. Como puede comprobarse, los campos más representados son los de la Biomecánica de Apoyo y de la Biomecánica Aplicada, con 107 y 98 tesis leídas, respectivamente y que representan el 45,9\% y $42,1 \%$ del total. A continuación, se encuentran las tesis leídas en el Campo de Biomecánica y Tecnologías, con 25 tesis, que representan un 10,7\% del total y, por último, las tesis leídas en el campo de la Biomecánica Básica, en las que se aborda el estudio de teorías explicativas del movimiento humano y deportivo, de carácter residual, con sólo tres tesis leídas que representa el $1,3 \%$ del total.

Tabla 2

Distribución de las tesis doctorales en función de los campos temáticos agrupados.

\begin{tabular}{lcc}
\hline Campos temáticos & $\mathrm{n}$ & $\%$ \\
\hline Biomecánica Básica & 3 & 1,3 \\
Biomecánica Aplicada & 98 & 42,1 \\
Biomecánica de Apoyo & 107 & 45,9 \\
Biomecánica-Tecnología & 25 & 10,7 \\
\hline Total & 233 & 100 \\
\hline
\end{tabular}

En la figura 2 se presenta la evolución en el tiempo de las tesis realizadas en cada uno de los campos temáticos, constatándose la existencia de una tendencia de crecimiento progresivo en los tres campos más representados. Esto es, en los campos de la Biomecánica de Apoyo que pasa de dos a 43 tesis realizadas entre el primero y cuarto periodo, la Biomecánica Aplicada que pasa de seis a 50 tesis, y la Biomecánica/Tecnologías que pasa de dos a 15 tesis. A pesar de estas tendencias, los resultados del análisis de contingencia demuestran que no existe una asociación estadísticamente significativa entre los periodos académicos y los campos temáticos $\left(X^{2}: 6.755\right.$ y $p:$.344)

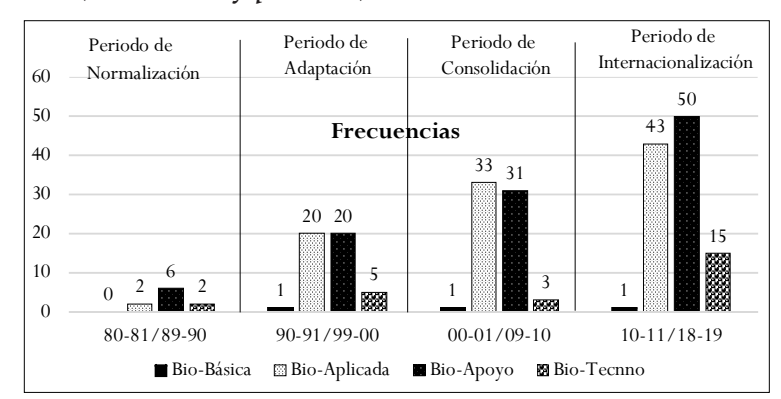

Figura 2. Evolución de la producción de tesis doctorales en función de los campos temáticos agrupados.

Para un análisis más pormenorizado, en las figuras tres, cuatro y cinco, se presenta la evolución que se ha producido en el número de tesis leídas en cada uno de los subcampos temáticos establecidos dentro de los campos temáticos principales. Concretamente, se presentan los datos de los tres campos temáticos más representados, como son la Biomecánica Aplicada, la Biomecánica de Apoyo, y la Biomecánica-Tecnologías.

En primer lugar, en el campo temático de la Biomecánica Aplicada (figura 3), que integra a las tesis cuya variable independiente es del ámbito de la biomecánica, se puede constatar que en los subcampos de Análisis de la Técnica Deportiva y de Análisis del Movimiento se produce una evolución que no es de crecimiento constante a lo largo del tiempo. En el primero, se leyeron un total de 35 tesis y en el segundo 32 tesis, lo que representan el 37,2\% y el 34\% del total de tesis en este campo temático. Por el contrario, en el subcampo de Análisis Varios, que integra a tesis realizadas en temáticas como la fuerza muscular, la coordinación, el desarrollo y elaboración de test de fuerza o coordinación, etc, se leyeron 27 tesis, que representa el $28,7 \%$ del total de tesis en este campo temático y en el que sí que se observa un crecimiento que es progresivo en el tiempo, pasando de 2 a 17 tesis entre el segundo y cuarto periodo. A pesar de estas tendencias, los resultados del análisis de contingencia vienen a confirmar que no existe una asociación estadísticamente significativa entre los periodos académicos y los subcampos temáticos de este grupo $\left(X^{2}: 12.290\right.$ y $p$ : .056).

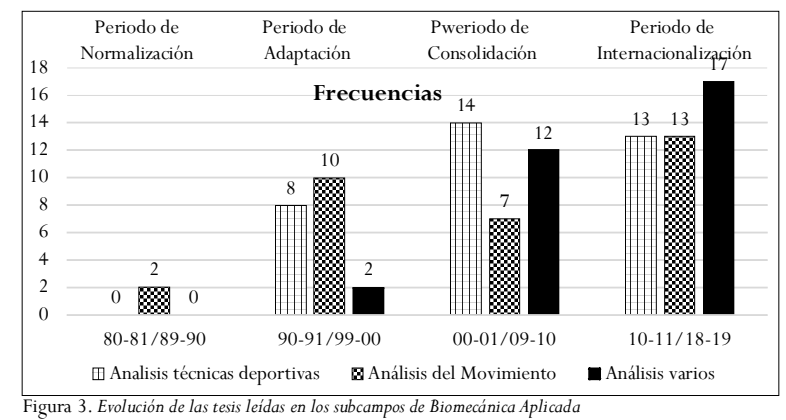

En segundo lugar, en el campo temático de la Biomecánica de Apoyo (figura 4), vinculado a las perspectivas científicas que abordan el ámbito deportivo y que integra a las tesis cuya variable independiente y objetivo concreto que se utiliza en el trabajo no responde a ninguna variable biomecánica, y en el que se puede constatar que el subcampo más representado y con un crecimiento constante es el del Área Médica con 73 tesis leídas que representa el 68,2\% del total del campo temático, con un crecimiento progresivo en el tiempo que pasa de tres a 31 tesis entre el primero y cuarto periodo. También en el subcampo de Fisiología con 11 tesis leídas que representan el 10\% del total del campo temático, se observa un crecimiento progresivo, aunque con valores muy inferiores al pasar de una a ocho tesis leídas entre el segundo y cuarto periodo, sin que en el primer periodo se hayan leído tesis referidas a este subcampo. En el subcampo de Control Motor con seis tesis leídas que representa el $56 \%$ del total del campo temático, la mayoría de las cuales fueron leídas en el tercer periodo (cuatro), sin que se produzca un crecimiento progresivo en el tiempo. A pesar de estas ten- 
dencias, los resultados del análisis de contingencia vienen a confirmar que no existe una asociación estadísticamente significativa entre los periodos académicos y los subcampos temáticos de este grupo $\left(X^{2}\right.$ : 11.311 y $p:$.255).

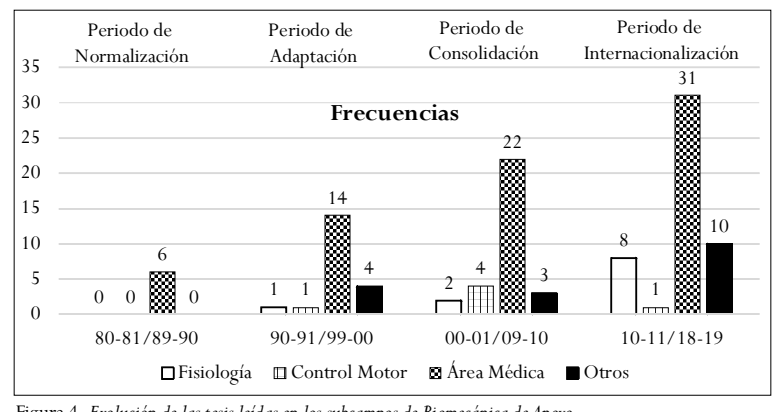

En tercer lugar, en el campo temático de la Biomecánica-Tecnología (figura 5), con tesis orientadas en los desarrollos biomecánicos que pueden utilizarse para registrar el movimiento, y en el que se constata que el subcampo más representado es el de la Fotogrametría con 14 tesis leídas, lo que representa el $43,7 \%$ del total de tesis en el campo temático que muestra un crecimiento progresivo del primer al tercer periodo pasando de 1 a 7 tesis realizadas, respectivamente, aunque con una caída importante en el cuarto periodo con tan sólo tres tesis leídas. El segundo subcampo más representado es el de las tesis realizadas sobre otras tecnologías (termografía, modelado, sensores inerciales, realidad virtual, robótica, resonancia magnética, etc,), con un total de 10 tesis que representan el 31,3\% del total en el campo temático, todas ellas leídas en el tercer y cuarto periodo y siendo en este último, el subcampo más representado de todos con nueve tesis. Por último, el subcampo de la Dinamometría/ Acelerometría, con ocho tesis leídas que representan el 25\% del total del campo temático y que muestra una evolución estable en el tiempo, esto es, entre una y tres tesis realizadas en cada uno de los periodos. En este caso, y dado el limitado número de tesis no se realizado el análisis de asociación mediante tablas de contingencia.

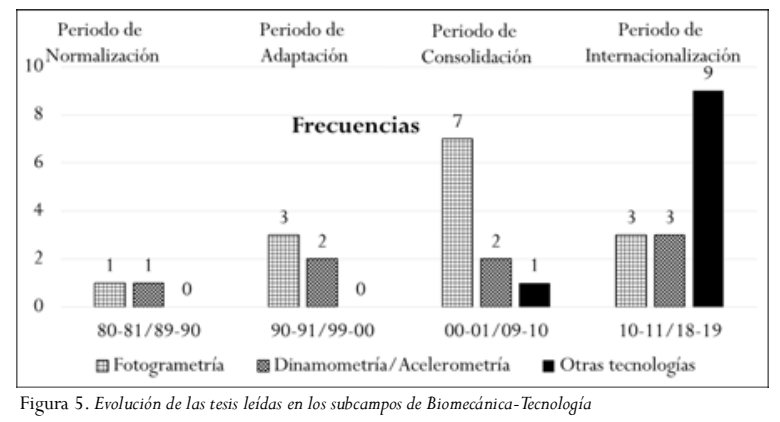

\section{Los términos más representados en los títulos} de las tesis

También se ha creído interesante extraer los términos más representados en los títulos de la tesis. En la tabla 3 se presentan los resultados obtenidos, constatándose que el término más representado en los títulos de las tesis y a distancia del resto, es el de la «marcha», con 61 apariciones, seguido de los términos «Análisis biomecánico/Estudio biomecánico», Cinemática/Cinemático», vinculados en la mayoría de casos al área del rendimiento deportivo, más propios de la orientación clásica de la Biomecánica Deportiva. A continuación, con una menor frecuencia aparecen términos como «Salto», «Carrera», «Rendimiento», «Entrenamiento», «Modelo», Lanzamiento»y «Muscular», vinculados al entrenamiento deportivo y al servicio dirigido al apoyo del entrenamiento de los deportistas, y otros términos como «Pie» o «Calzado», vinculados al área médica/rehabilitación y de los materiales.

En un análisis más en profundidad sobre el término de la «marcha»se ha comprobado que las 61 apariciones en los títulos de tesis se vinculan con tesis realizadas en Áreas Médicas (68,8\% del total), seguido de las Ingenierías (11\% del total), y de la Educación Física y Ciencias del Deporte ( $8 \%$ del total).

\begin{tabular}{lc} 
Tabla 3 & \\
Relación de los términos más representados en los títulos de las tesis & \\
\hline Términos más representados en los títulos de las tesis & $\mathrm{n}$ \\
\hline Marcha & 61 \\
Análisis biomecánico/Estudio biomecánico & 31 \\
Cinemático/cinemática & 26 \\
Pie & 21 \\
Salto & 18 \\
Carrera & 18 \\
Pacientes & 17 \\
Rendimiento & 14 \\
Calzado & 10 \\
Entrenamiento & 9 \\
Modelo & 8 \\
Lanzamiento & 7 \\
Muscular & 7 \\
\hline
\end{tabular}

\section{Discusión}

Ante la falta de referencias previas en la temática estudiada, consideramos que este trabajo tiene la utilidad de establecer un diagnóstico objetivo sobre la forma en que ha evolucionado la investigación en la Biomecánica Deportiva en los últimos 40 años.

Los datos vienen a confirmar que los campos temáticos en los que mayor número de tesis se han realizado son los de la Biomecánica de Apoyo y de la Biomecánica Aplicada, ambos con unas trayectorias de crecimiento constante desde el primer periodo analizado y con una relación bastante equilibrada entre ambos. Una circunstancia que no es extraña en la medida que representan a dos campos de investigación que acogen las orientacio- 
nes más extendidas en la Biomecánica Deportiva. Por un lado, la Biomecánica de Apoyo, donde a través de técnicas biomecánicas se analiza un entrenamiento concreto, un programa para la salud, una variable sociológica, conductual, psicológica etc, ... desde las perspectivas de la Fisiología, el Control Motor, el Área Médica, u otros áreas que, en su desarrollo, han ido creciendo a lo largo del tiempo hasta acabar siendo el campo temático más representado en el último periodo como una muestra clara de la forma en que ha evolucionado la investigación en esta disciplina científica. Por otro lado, la Biomecánica Aplicada, dirigida básicamente al análisis biomecánico de la técnica deportiva y del movimiento humano. En suma, los datos muestran los cambios que se han producido en los campos temáticos donde, el interés de otras perspectivas por la biomecánica (Biomecánica de Apoyo) ha experimentado un crecimiento comparable al de la Biomecánica Aplicada.

Probablemente la forma en que han evolucionado las temáticas y contenidos de las tesis doctorales en el campo de la Biomecánica Deportiva esté asociado al desarrollo y evolución de los primeros proyectos de investigación de carácter competitivo que se pusieron en marcha desde el año 1990. Así, el Plan Nacional de Investigación Científica y Desarrollo Tecnológico (PN I+D) del 1988, supuso la primera referencia y origen.

En el año 1988 se creó el Plan Nacional de Investigación Científica y Desarrollo Tecnológico (PN I+D), lo que constituye la primera referencia y el origen de los programas específicos de I+D en el deporte. Dicho PN I+D estuvo vigente entre los años 1988 y 1991. En este primer período, los recursos del Consejo Superior de Deportes (CSD) se sumaron a los aportados por el Plan Nacional de I+D con el objetivo de cubrir las necesidades derivadas de la organización de la Olimpiada de Barcelona'92.

En el año 1991 se abrió un nuevo período que lanzó el II Plan Nacional de I+D (1992-1995) en el que la investigación en Ciencias del Deporte quedó encuadrada en el Programa sectorial de Salud y Farmacia. La razón que justificaba esta adscripción era que la mayoría de los proyectos desarrollados en Ciencias del deporte correspondían al campo de la Fisiología y de la Medicina del Deporte (Vera \& Hernández, 1998). Ciertamente, en los inicios de la década de los años 90 el peso de los Centros y Facultades de CAFD y del área Educación Física Deportiva era reducido en sus capacidades de promover investigación.

Por otro lado, y en un ámbito más general, hay que tener en cuenta también la evolución positiva que ha experimentado el sector deportivo en España. Durante el periodo 1980-2010 la práctica deportiva de la ciudadanía creció un 15\%, pasando del 25\%, al 40\% respectivamente (García Ferrando \& Llopis, 2011). En el segundo trimestre de 1991 el mercado de trabajo en los sectores de entrenamiento, docencia, animación y dirección de instalaciones era de 42.679 empleos frente a los 9.824 existentes en 1973 (Martínez del Castillo, Puig, Fraile, \& Boixeda, 1991). También, la década de los años 90 fue decisiva para el crecimiento e influencia del sector deportivo sobre la economía. El impacto del consumo del deporte en el PIB pasó del 1,2\% en 1991, a una previsión de 2,38\% para 2006 (Alonso, Ruesga, Sáez, \& Vicens, 1991; Lera, Rapún, \& Gárate, 2005).

Este impulso y cambio de tendencia que se daba en el sector deportivo y en la promoción y reconocimiento de los valores y efectos de la actividad física y del deporte como un medio de cambio social, se produjo también de forma paralela en el ámbito de la investigación en el deporte. De hecho, el periodo comprendido entre los años 1990 y 2000 fueron claves, gracias al impulso recibido de las convocatorias, tanto del CSD, como de los Planes Nacionales de Investigación I+D+i. Este hecho fue aprovechado por los grupos de investigación de las Facultades de CAFD que comenzaron a liderar proyectos de forma coordinada, en ocasiones junto a las Federaciones Deportivas, tanto Nacionales, como Internacionales, con el objetivo inicial de la mejora del entrenamiento y seguimiento de los deportistas. Un ejemplo de ello fue el Proyecto Coordinado de Análisis Biomecánico de los Lanzamientos y de las Carreras de Velocidad en el Campeonato del Mundo de Atletismo Sevilla'99, financiado por la Comisión Interministerial de Ciencia y Tecnología (CICYT), dirigido por el profesor Dr. Marcos Gutiérrez Dávila de la Universidad de Granada y en el que participaron grupos de la Facultad de CAFD de la Universidad de Valencia, del INEF de León, del INEF de Lleida, de la Facultad de CAFD de Granada, junto con la Unidad de Biomecánica del CNICD de Madrid

Como referencia del cambio de tendencias y del protagonismo que fueron ganando los INEFs y Facultades de CAFD en el desarrollo de proyectos de investigación competitivos, en la figura 6 se presenta la distribución de los proyectos financiados por el Centro de Alto Rendimiento y Ciencias del Deporte (CARICD) por áreas de conocimiento en dos periodos clave como son el de normalización académica y adaptación a los paradigmas Universitarios. Como se puede comprobar, durante el período comprendido entre 1988 y 1994 las 
áreas de Medicina e Ingeniería obtuvieron más de la mitad de los proyectos financiados $(25,2 \%$ y $25,3 \%$ respectivamente), mientras que los INEF y Facultades de Ciencias de la Actividad Física y del Deporte (CAFD) sólo lideraron el 5\% de ellos. Por el contrario, durante el período comprendido entre 1995 y 1997, se produce un cambio de tendencia importante por cuanto las áreas de Medicina e Ingeniería descendieron en su capacidad de liderar proyectos hasta el 35\% del total, mientras que los INEF y Facultades de CAFyD, aumentaron su cuota de liderazgo llegando prácticamente al 20\% del total de proyectos financiados, por encima del área de Ingenierías y muy cerca de la de Medicina.

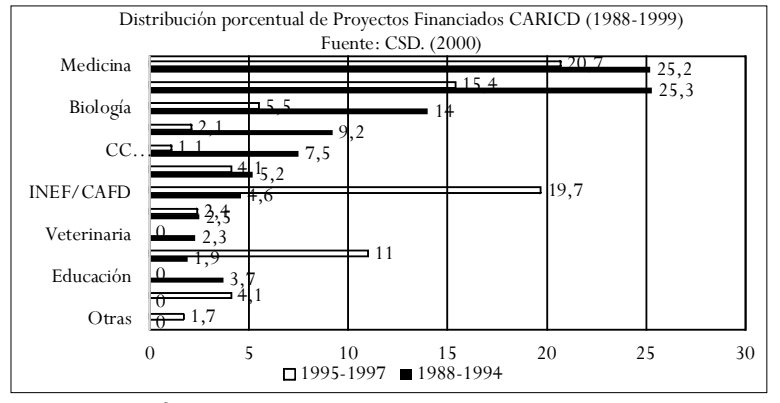

Figura 6. Proyectos financiados por el CARICD (1988-1997). Fuente. CSD.

En cuanto a las tesis en el campo de la Biomecánica Aplicada, la evolución de sus temáticas viene a demostrar la influencia que tuvieron sobre ellas el desarrollo de los proyectos realizados entre los años 1990 y 2010, lo que probablemente provocó que la mayoría de las tesis en dichos periodos se realizaran en los subcampos del Análisis Biomecánico de la Técnica Deportiva y en el del Análisis Biomecánico del Movimiento Humano. Como muestra de la evolución positiva que se estaba produciendo en el campo de la Biomecánica Deportiva, baste señalar que entre los años 2005 y 2011, de los 192 proyectos de investigación financiados por el Plan Nacional, 31 de ellos eran de Biomecánica, lo que suponía el 16\% del total (Ferro, 2012). A partir del año 2000, emerge una nueva tendencia con orientaciones más abiertas temáticas, al incorporar trabajos centrados en la fuerza muscular, la coordinación, o la elaboración de test de fuerza, entre otros temas, y que en este trabajo hemos denominado como el subcampo de «Análisis Varios», que en el último periodo entre los años 2010 y 2019, acabó convirtiéndose en el mayoritario con un total de 17 tesis leídas.

Por otra parte, en lo que afecta al campo de la Biomecánica de Apoyo, el liderazgo en la producción de tesis lo ostenta el subcampo del Área Médica, que evoluciona de forma creciente y progresiva en el tiempo, demostrándose su relevante papel en el desarrollo de la
Biomecánica Deportiva. Las tesis realizadas desde otras disciplinas científicas como la Fisiología, el Control Motor, y otros como la Ergonomía, o el Diseño Industrial, con objetivos de estudio sobre materiales, vestuario, calzado, confort, instalaciones, muestran también un crecimiento progresivo en el tiempo, aunque menor y anotando su mayor incremento en el último periodo. Un hecho que revela un cierto cambio de tendencias en la investigación en la Biomecánica de Apoyo.

Por último, el desarrollo del campo temático de Biomecánica-Tecnologías, que ha estado tutelado fundamentalmente por las Ingenierías y Centros vinculados a ellas. La evolución en este campo está caracterizada por la presencia de la Fotogrametría como el subcampo en el que más tesis se han realizado en los tres primeros periodos. Un hecho que no es extraño por cuanto la Fotogrametría es una técnica de registro básica para la realización de cualquier estudio de biomecánica. Sin embargo, en el cuarto periodo, esta tendencia se rompe, de manera que la mayoría de las tesis se realizan sobre lo que se denomina «Otras Tecnologías», en las que están: la termografía, sensores inerciales, realidad virtual, robótica, o la resonancia magnética entre otras. Probablemente, el menor uso de la fotogrametría en este periodo de internacionalización esté asociado a la complejidad de estas técnicas y al escaso interés por la descripción del movimiento, orientándose más hacia los aspectos explicativos. En suma, una muestra del aprovechamiento y utilización de nuevas tecnologías que ha permitido a los investigadores abordar en mejores condiciones el estudio de realidades más complejas a pesar de que el perfil de formación del estudiantado de CAFD debería incorporar una mayor orientación tecnológica basada en la enseñanza de habilidades de desarrollo (Carreiro da Costa, González Valeiro, \& González Villalobos, 2016).

También ha resultado interesante constatar que el término más utilizado en los títulos de las tesis sea el de la «Marcha» como objeto de estudio. Un término que posiciona a la Marcha Humana, como un tema transversal que ha sido abordado desde diferentes perspectivas, tanto dese el área médica, como de las Ingenierías, como de la de la Educación Física y las CAFD y vinculado a los tres campos temáticos considerados, la Biomecánica de Apoyo, la Aplicada y la unida a las Tecnologías. Los estudios realizados sobre la biomecánica de la marcha han permitido, de hecho, que profesionales de la salud y de la educación física conozcan y puedan prescribir los ejercicios más eficaces para el tratamiento de sus pacientes (Cadenas-Sánchez, Arellano, \& López 
Contreras, 2015). En suma, un campo de estudio muy amplio al estar vinculado a la salud, la rehabilitación, la creación de nuevos sistemas de medida, y al rendimiento deportivo.

\section{Conclusiones}

Los resultados de este estudio vienen a constatar que la Biomecánica Deportiva ha sido prolífica en su capacidad de promover tesis doctorales con un crecimiento constante y progresivo desde el año 1983. Una evolución que ha corrido paralela al crecimiento de la investigación en esta disciplina científica gracias al trabajo realizado por laboratorios y unidades de investigación universitarias.

También ha quedado constatado que los dos campos más representados en la realización de tesis doctorales son los de la Biomecánica de Apoyo y de la Biomecánica Aplicada. El primero, vinculado a estudios cuya variable independiente y objetivo concreto que se utiliza no responde a ninguna variable biomecánica, esto es, cuando se evalúa un entrenamiento concreto, un programa para la salud, una variable sociológica, conductual, psicológica etc, y el segundo, a estudios cuya variable independiente es del ámbito de la biomecánica, dentro de la orientación clásica de la Biomecánica Deportiva. Unos datos que refuerzan el carácter aplicado de la investigación en este campo y que dejan a la Biomecánica Básica, orientada en las teorías explicativas, como un campo de investigación residual.

Estas tendencias vienen también a demostrar que la Biomecánica deportiva ha ido creciendo y ampliando su campo de acción en ámbitos orientados a la salud y a la ergonomía, al encontrar los investigadores mayor masa crítica, mejores condiciones y financiación para estos campos en las convocatorias de ayudas Nacionales y/o Europeas.

Con todo, y a pesar de esta tendencia de crecimiento hacia el campo de la salud, la Biomecánica Deportiva ha seguido manteniendo una presencia importante en el apoyo y desarrollo del deporte y de los deportistas de competición evolucionando y conformando equipos de investigación integrados por profesionales provenientes del campo del deporte, de las tecnologías, de la salud y de la ingeniería.

\section{Referencias}

Alonso, J., Ruesga, S., Sáez, F., \&Vicens, J. (1991). Impacto económico del deporte en España, Revista de Investigaciones y Documentación sobre las Ciencias de la Educación Física y el deporte, 18 ,
22-35

COLEF. (2021). Formación universitaria en ciencias de la actividad física y el deporte. Recuperado de https://www.consejocolef.es/formacion-universitaria

Cadenas-Sánchez, C., Arellano, R., \& López Contreras, G. (2015). Revisión de la biomecánica de la marcha en medio acuático vs. Terrestre. Retos, 28, 128-133

Carreiro da Costa, F., GonzálezValeiro, M., \& GonzálezVillalobos, M. (2016). Innovación en la formación del profesorado de educación física. Retos, 29, 251-257

Delgado, M.A., \& Medina, J. (1997). Investigación sobre las ciencias de la actividad física y el deporte en la universidad española.1981-1996. Motricidad. European Journal of Human Movement, 3, 131-150

Ferro,A. (2012). Tendencia en I+D+I en Biomecánica aplicada al deporte. RICYDE. Revista internacional de ciencias del deporte, 8 (30), 275. doi:10.5232/ricyde2012.030

Fuentes, E., \& Argimbau, L. (2010). Las tesis doctorales en España (1997-2008): análisis, estadísticas y repositorios cooperativos. Revista Española de Documentación Científica, 33(1), 63- 89. https://doi.org/10.3989/redc.2010.1.711

García Ferrando, \& M., Llopis, R. (2011). Ideal democrático y bienestar personal. Encuesta sobre los hábitos deportivos en España 2010. CIS, CSD, Madrid

García García, J. M. (2016). Evolución de las tesis doctorales en Educación Física y Deportes en España (1990-2013). Apunts. Educación Física y Deportes, 125, 21-34. https://doi.org/ 10.5672/apunts.2014-0983.es.(2016/3). 125.02

Lera, F., Rapún, M., \& Gárate, J. (2005). Aproximación al gasto total en deporte. En CSD, Análisis y evaluación económica de la participación deportiva en España, 51, 121-172

López Fernández, I., \& Almendral, P. (2001). Contenido de los planes de estudio de la licenciatura en Ciencias de la actividad Física y del Deporte en España. Apunts. Educación Física y Deportes, 65, 72-85.

Martínez del Castillo, J., Puig, N., Fraile,A., \& Boixeda, A. (1991). Estructura ocupacional del deporte. Encuesta en los sectores de entrenamiento, animación, docencia y dirección. En CSD, Estructura Ocupacional y mercado laboral en el Deporte, 4, 77-128

Michiyoshi Ae,Yasushi Enomoto, Norihisa Fujii, \& HidekiTakagi (ed) (2016). Proceedings of the XXXIV International Conference of Biomechanics in Sport. ISBS, Tsukuba (Japan)

Ministerio de Ciencia y Tecnología. (1999). Plan Nacional de Investigación Científica, Desarrollo e Innovación Tecnológica (2000-2003). Comisión Interministerial de Ciencia y Tecnología, Madrid

Ministerio Universidades. (2020). Datos y cifras del Sistema Universitario Español. Publicación 2019-20. Secretaría General de Universidades, Madrid

Pastor Pradillo, J.L. (2003). La investigación en las Ciencias de la Actividad Física y el Deporte. Perspectiva histórica. Ágora para la educación física y el deporte, 2-3, 39-50.

Ponce de León Elizondo, A., Gargallo, E., \& Loza. E. (1998). Anàlisi de les tendencies a les tesis doctorals d'educació física. Cursos 1980-81 a 1995-96. Apunts, 52, 104-108.

Vera. P., \& Hernández, J.L. (coord.) (1998). Libro Blanco I+D en el Deporte. Ministerio de Educación y Cultura, Ministerio de Industria y Energía, Madrid 